\title{
ANOTHER CHARACTERIZATION OF BLO
}

\author{
COLIN BENNETT ${ }^{1}$
}

\begin{abstract}
It is shown that a locally integrable function $f$ on $\mathbf{R}^{n}$ has bounded lower oscillation ( $f \in \mathrm{BLO}$ ) if and only if $f=M F+h$, where $F$ has bounded mean oscillation ( $F \in \mathrm{BMO}$ ) and $M F<\infty$ a.e., and $h$ is bounded. Here, $M F$ is a variant of the familiar Hardy-Littlewood maximal function: $M F=\sup _{Q \ni x} Q(F)$ (no absolute values), where $Q(F)$ is the mean value of $F$ over the cube $Q$.
\end{abstract}

We consider real-valued locally integrable functions $f$ on $\mathbf{R}^{n}$. When $Q$ is a cube in $\mathbf{R}^{n}$ with sides parallel to the coordinate axes, we denote by $Q(f)$ the mean value of $f$ over $Q$. Define a maximal function $M f$ of $f$ by .

$$
(M f)(x)=\sup _{Q \ni x} Q(f)=\sup _{Q \ni x} \frac{1}{|Q|} \int_{Q} f(y) d y \quad\left(x \in \mathbf{R}^{n}\right),
$$

where the supremum extends over all cubes $Q$ that contain $x$. Note that $f$ is not assumed to be nonnegative so that $M f$ may take on negative values. However, it follows from the differentiation theorem that $M f \geqslant f$ a.e. Moreover, it is clear $|M f|$ is dominated by $M|f|$, the latter being the familiar Hardy-Littlewood maximal function of $f$, and so, in particular, the maximal operator $M$ is bounded on $L^{2}$. We shall make use of the obvious fact that if $Q$ and $Q^{\prime}$ are two cubes with $Q \subset Q^{\prime}$, then $Q^{\prime}(f)$ is dominated by $(M f)(x)$ for every $x$ in $Q$, and so

$$
Q^{\prime}(f) \leqslant \inf _{Q} M f \quad\left(Q \subset Q^{\prime}\right) .
$$

A locally integrable function $f$ on $\mathbf{R}^{n}$ is said to be of bounded mean oscillation $(f \in \mathrm{BMO})$ if

$$
\|f\|_{\text {BMO }}=\sup _{Q} Q(|f-Q(f)|)
$$

is finite. The space BMO is a linear space and, when the constant functions are factored out, the functional in (2) is a norm under which BMO is a Banach space. It is well known (cf. [3, p. 227]) that replacing (2) by the corresponding quadratic functional results in an equivalent norm on BMO. In particular, there is a constant $c$, depending only on the dimension $n$, such that

$$
\left(\frac{1}{|Q|} \int_{Q}|f(x)-Q(f)|^{2} d x\right)^{1 / 2} \leqslant c\|f\|_{\mathrm{BMO}}
$$

for every cube $Q$ in $\mathbf{R}^{n}$.

Received by the editors July $30,1981$.

AMS (MOS) subject classifications (1970). Primary 44A25.

${ }^{1}$ Research supported in part by NSF Grant MCS-8102194.

C1982 American Mathematical Society $0002-9939 / 81 / 0000-0799 / \$ 02.25$ 
Recently, R. R. Coifman and R. Rochberg [2] have introduced the space BLO of functions of bounded lower oscillation. The space is defined analogously to BMO except that in (2) one subtracts from $f$ the essential infimum

$$
f_{Q}=\underset{x \in Q}{\operatorname{essinf}} f(x)
$$

instead of the mean value $Q(f)$. Thus, a locally integrable function $f$ on $\mathbf{R}^{n}$ is in BLO if

$$
\|f\|_{\mathrm{BLO}}=\sup _{Q}\left(Q(f)-f_{Q}\right)
$$

is finite. The BLO property need not be preserved under multiplication by negative constants so, in particular, BLO is not a linear space, and, despite the notation, the functional in (4) is not a norm. It is easy to check, however, that $\|\cdot\|_{\text {BLO }}$ is subadditive and positive-homogeneous.

Note that every $L^{\infty}$-function is in BLO, with

$$
\|f\|_{\mathrm{BLO}} \leqslant 2\|f\|_{L^{\infty}} \quad\left(f \in L^{\infty}\right),
$$

and every BLO-function is in BMO, with

$$
\|f\|_{\mathrm{BMO}} \leqslant 2\|f\|_{\mathrm{BLO}} \quad(f \in \mathrm{BLO}) .
$$

Using a representation theorem of Carleson for BMO-functions, Coifman and Rochberg [2] have shown that every BMO-function is representable as the difference of two BLO-functions. Moreover, they have shown that BLO-functions arise, modulo bounded functions, as logarithms of maximal functions. In other words, a locally integrable function $f$ belongs to BLO if and only if

$$
f=\alpha \log M F+h,
$$

where $\alpha$ is a nonnegative constant, $F$ is a nonnegative locally integrable function whose maximal function $M F$ is finite a.e., and $h$ is an $L^{\infty}$-function.

Let us note also the result of [1] that if $f \in \mathrm{BMO}$ (and has finite maximal function), then the decreasing rearrangement $f^{*}$ (of $|f|$ ) is in BLO; in fact, this condition characterizes the rearrangements of BMO-functions.

In this note, we shall obtain a different description of BLO-functions. We shall show that they arise, modulo bounded functions, as the maximal functions $M F$ of BMO-functions $F$. Of course, we must exclude those $F$ for which $M F$ is identically infinite.

We shall need two lemmas, the first of which may be regarded as a refinement of Theorem 4.2 in [1].

LEMMA 1. If $F \in B M O$ and if $Q$ is any cube in $\mathbf{R}^{n}$, then

$$
Q(M F) \leqslant c\|F\|_{B M O}+\inf _{Q} M F,
$$

where $c$ is a constant depending on the dimension $n$. In particular, if MF is not identically infinite, then $M F \in B L O$ and

$$
\|M F\|_{B L O} \leqslant c\|F\|_{B M O} .
$$


Proof. Fix $Q$ and let $\bar{Q}$ be the cube concentric to $Q$ with dimensions three times as large. Write

$$
F=(F-\bar{Q}(F)) \chi_{\bar{Q}}+\left[\bar{Q}(F) \chi_{\bar{Q}}+F \chi_{\bar{Q}} c\right]=G+H
$$

say.

By the Cauchy-Schwarz inequality,

$$
Q(M G) \leqslant\left(\frac{1}{|Q|} \int_{Q}(M G)^{2} d x\right)^{1 / 2} \leqslant|Q|^{-1 / 2}\|M G\|_{L^{2}\left(\mathbf{R}^{n}\right)}
$$

But $M$ is bounded on $L^{2}$, so using the definition of $G$, the fact that $|\bar{Q}|=3^{n}|Q|$, and (3), we obtain

$$
Q(M G) \leqslant c\|F\|_{\text {BMO }}
$$

Next we shall show that

$$
Q(M H) \leqslant c\|F\|_{\mathrm{BMO}}+\inf _{Q} M F,
$$

which, together with (10) and the fact that $F=G+H$, will establish the desired result (8). In order to establish (11), it will suffice to show that $M H(x)$ is dominated by the right-side of (11) for every $x \in Q$, and for this it will be enough to show that

$$
P(H) \leqslant c\|F\|_{\text {BMO }}+\inf _{Q} M F
$$

for every cube $\boldsymbol{P}$ in $\mathbf{R}^{n}$ containing $\boldsymbol{x}$.

The result follows directly from (1) if $P$ does not meet $\bar{Q}^{c}$, for then $H=\bar{Q}(F)$ on $P$ and so $P(H)=\bar{Q}(F) \leqslant \inf _{Q} M F$. So suppose $P \cap \bar{Q}^{c} \neq \varnothing$, and let $P^{\prime}$ be the smallest cube containing both $P$ and $\bar{Q}$. Since $P$ contains the point $x$ of $Q$, it is clear that

$$
\left|P^{\prime}\right| \leqslant c|P|
$$

for some constant $c$ depending only on the dimension $n$. Furthermore, from the definition of $H$,

$$
\begin{aligned}
\int_{P}\left(H-P^{\prime}(F)\right) & \leqslant \int_{P^{\prime}}\left|H-P^{\prime}(F)\right|=\int_{\bar{Q}}\left|\bar{Q}(F)-P^{\prime}(F)\right|+\int_{P^{\prime} \cap \bar{Q}^{\varphi}}\left|F-P^{\prime}(F)\right| \\
& \leqslant\left(\int_{\bar{Q}}+\int_{P^{\prime} \cap \bar{Q}}\left|F-P^{\prime}(F)\right|=\int_{P^{\prime}}\left|F-P^{\prime}(F)\right|,\right.
\end{aligned}
$$

so, using (2) and (13), we obtain $P\left(H-P^{\prime}(F)\right) \leqslant c\|F\|_{\mathrm{BMO}}$. Since $P^{\prime} \supset Q$, an appeal to (1) now gives

$$
P(H)=P\left(H-P^{\prime}(F)\right)+P^{\prime}(F) \leqslant c\|F\|_{\mathrm{BMO}}+\inf _{Q} M F .
$$

This establishes (12) and hence, as we remarked above, completes the proof of (8).

It is now clear from (8) that if $(M F)(x)$ is finite at any one point $x$ in $\mathbf{R}^{n}$, then $(M F)(y)$ is finite a.e. on every cube $Q$ containing $x$, hence on all of $\mathbf{R}^{n}$. In that case, we may subtract inf ${ }_{Q} M F$ from each side of (8) and take the supremum over all $Q$ to obtain (9). 
We require one further lemma.

LEMMA 2. A locally integrable function $f$ on $\mathbf{R}^{n}$ belongs to $B L O$ if and only if $M f-f$ belongs to $L^{\infty}$. Furthermore,

$$
\|M f-f\|_{L^{\infty}}=\|f\|_{B L O} .
$$

Proof. Suppose first that $f$ belongs to BLO. Let $x$ be any Lebesgue point of $f$ and let $Q$ be any cube containing $x$. Then $f(x) \geqslant f_{Q}$ and so

$$
Q(f)-f(x) \leqslant Q(f)-f_{Q} \leqslant\|f\|_{\text {BLO }}
$$

Taking the supremum over all cubes $Q$ containing $x$, and then the supremum over all Lebesgue points $x$ of $f$, we find that $M f-f$ belongs to $L^{\infty}$ and

$$
\|M f-f\|_{L^{\infty}} \leqslant\|f\|_{\text {BLO. }}
$$

Conversely, suppose $M f-f$ belongs to $L^{\infty}$ and let $Q$ be any cube in $\mathbf{R}^{n}$. Any point $x$ in $Q$ for which

$$
f(x)<Q(f)-\|M f-f\|_{L^{\infty}}
$$

must satisfy

$$
(M f)(x)-f(x) \geqslant Q(f)-f(x)>\|M f-f\|_{L^{\infty}},
$$

and consequently such points $x$ constitute a set of measure zero. Hence the essential infimum $f_{Q}$ is at least as large as the value on the right-hand side of (16), and so

$$
Q(f)-f_{Q} \leqslant\|M f-f\|_{L^{\infty}} .
$$

Taking the supremum over all $Q$, we obtain the reverse inequality to (15), and hence (14) is established.

Our main result is as follows.

THEOREM. A locally integrable function $f$ on $\mathbf{R}^{n}$ belongs to $B L O$ if and only if there are functions $h$ in $L^{\infty}$ and $F$ in $B M O$ with $M F$ finite a.e. such that

$$
f=M F+h \text {. }
$$

Furthermore,

$$
\|f\|_{B L O} \sim \inf \left(\|F\|_{B M O}+\|h\|_{L^{\infty}}\right),
$$

where the infimum extends over all representations of the form (17).

Proof. If $f$ has a representation as in (17), then $M F$ belongs to BLO by virtue of Lemma 1 . Since $h$ is bounded, hence in BLO, we see that $f$ is in BLO. Furthermore, from (5) and (9),

$$
\|f\|_{\mathrm{BLO}} \leqslant\|M F\|_{\mathrm{BLO}}+\|h\|_{\mathrm{BLO}} \leqslant c\|F\|_{\mathrm{BMO}}+2\|h\|_{L^{\infty}},
$$

so taking the infimum over all representations (17) of $f$, we obtain

$$
\|f\|_{\mathrm{BLO}} \leqslant c \inf \left(\|F\|_{\mathrm{BMO}}+\|h\|_{L^{\infty}}\right) \text {. }
$$

Conversely, if $f \in \mathrm{BLO}$, then $M f$ is finite a.e., by Lemma 2. Furthermore, by the same lemma, the function $f-M f$ is bounded, and so $f=M f+(f-M f)$ is a 
representation of the form (17) with $F=f$ and $h=f-M f$. Moreover, from (6) and (14),

$$
\|F\|_{\mathrm{BMO}}+\|h\|_{L^{\infty}}=\|f\|_{\mathrm{BMO}}+\|f-M f\|_{L^{\infty}} \leqslant 3\|f\|_{\mathrm{BLO}}
$$

so, combining this observation with (19), we establish (18).

REMARKS. The above analysis can also be carried through for a cube $Q_{0}$ instead of all of $\mathbf{R}^{n}$. In this case, BLO-functions on $Q_{0}$ are bounded below and so, by addition of suitable constants, can be rendered nonnegative. In this case, one obtains a result of the form (17) with $M$ the usual Hardy-Littlewood maximal function $\sup _{Q} Q(|f|)$.

I wish to thank my colleagues, Professors R. A. DeVore, W. Nestlerode, and R. Sharpley, for some interesting discussions concerning this material.

\section{REFERENCES}

1. C. Bennett, R. A. DeVore and R. Sharpley, Weak- $L^{\infty}$ and BMO, Ann. of Math. (2) 113 (1981), $601-611$.

2. R. R. Coifman and R. Rochberg, Another characterization of BMO, Proc. Amer. Math. Soc. 79 (1980), 249-254.

3. C. Sadosky, Interpolation of operators and singular integrals, Dekker, New York, 1979.

Department of Mathematics and Statistics, University of South Carolina, Columbia, South CAROLINA 29208 